\title{
The thinking doctor: clinical decision making in contemporary medicine
}

\author{
Authors: Michael Trimble ${ }^{A}$ and Paul Hamilton ${ }^{B}$
}

Diagnostic errors are responsible for a significant number of adverse events. Logical reasoning and good decision-making skills are key factors in reducing such errors, but little emphasis has traditionally been placed on how these thought processes occur, and how errors could be minimised. In this article, we explore key cognitive ideas that underpin clinical decision making and suggest that by employing some simple strategies, physicians might be better able to understand how they make decisions and how the process might be optimised.

KEYWORDS: Error, thinking, decision making, metacognition, bias

\section{Introduction}

The work of a surgeon is done in the operating theatre. Much time and effort is invested in skills training and great attention is paid to the operating environment. In contrast, the work of the physician is the decision-making process on the ward round. ${ }^{1}$ There is often little instruction about the decision-making process and its dangers, and often, little attention is paid to the environment where the decision-making process occurs. In the USA, it has been estimated that 44,000-98,000 patients die each year through medical error, ${ }^{2}$ and in the Harvard Medical Practice Study, diagnostic errors accounted for $17 \%$ of all adverse events. ${ }^{3}$

Thinking is core to the work of the physician. For American physician and philosopher Edmund Pellegrino, the clinical encounter defines medical practice. It can be viewed as a series of three questions: 'What is the problem?', 'What are the possible solutions?' and 'What is the best solution for this patient? ${ }^{4}$ In order to answer these questions, one needs to be able to reason and evaluate. It is our purpose in this article to explore this process, to consider some of the errors that may be encountered, and to offer some suggestions as to how we can avoid them.

\section{How do we make clinical decisions?}

Clinical diagnosis is more akin to the work of a detective than a scientist. Looking at the diagnostic process in more detail we

Authors: ${ }^{\text {A }}$ clinical lecturer, Centre for Medical Education, Queen's University Belfast and honorary consultant physician, Belfast Health and Social Care Trust, Belfast, UK; ${ }^{B}$ specialty registrar, Department of Clinical Biochemistry, Belfast Helath and Social Care Trust and honorary lecturer, Queen's University Belfast, Belfast, UK can break it down into the following steps:

$>$ information gathering

$>$ hypothesis generation

$>$ hypothesis testing

$>$ reflection.

Often the term 'hypothetico-deductive' is used to describe this process. Sir Arthur Conan Doyle was clearly fond of the idea of deductive reasoning and used the phrase 'the science of deduction' as chapter headings twice in the Sherlock Holmes stories. However, what happens in practice is that physicians seek 'an explanation of a particular fact by finding some salient features of the particular that allow it to be explained by some more general causative principles,' a process that the American philosopher Charles Sanders Peirce described as 'abduction'. The following simple examples adapted from Rapezzi, Ferrari and Branzi may help to clarify what is meant by the terms. ${ }^{6}$

Deduction is reasoning from the general to the particular:

$>$ All the marbles in the jar are white.

$>$ These marbles are from the jar.

$>$ Therefore these marbles must be white.

Induction is reasoning from the particular to the general:

$>$ These marbles have come from the jar.

$>$ These marbles are white.

$>$ Therefore the marbles in the jar are white.

Abduction is more of an inference, an informed guess that fits with the known facts:

$>$ The marbles in the jar are white.

$>$ These marbles are white.

> Therefore these marbles have come from the jar.

\section{Data gathering}

The first step in the process is information gathering. To quote Holmes it is a capital mistake to theorise before one has data. Insensibly one begins to twist facts to suit theories, instead of theories to suit facts. ${ }^{7}$ Carl Trueman notes that history is 'the remembered past' and, as such, is inevitably shaped by those who do the remembering. ${ }^{8}$ Patients attempt to understand their symptoms, interpreting them within their own frame of reference. Also, it is easy to influence the patient by the use of leading questions. As a story is rehearsed and repeated it may unintentionally change and so it becomes harder to establish 
the true course of events. We must be careful that we have the appropriate data before attempting to draw conclusions. As with the history, we must pay attention as we examine the patient. In A scandal in Bohemia, Sherlock Holmes chides Dr Watson 'You see, but you do not observe'. ${ }^{9}$ We can be blinkered in our perceptions and, if we are not actively seeking information, may miss important findings.

\section{Hypothesis generation}

We all use a selection of strategies to produce a list of differential diagnoses; varying them depending on the degree of familiarity with the condition and the severity of the patient's illness. Canadian emergency physician Pat Croskerry has done much work in this area and lists the following common approaches. ${ }^{10}$

\section{Pattern recognition}

At its most simplistic level, pattern recognition may seem easyas you hear the first lines of a history, familiar patterns become apparent: headache with neck stiffness and photophobia - and already the options are starting to line up. Add thunderclap onset (or fever) and our pattern is complete. But what of the cases where the pattern is incomplete - possibly because we have not observed all findings - or if the pattern is unfamiliar? Pattern recognition also depends on practice; for an experienced clinician, the complex case may seem to fit a pattern. But we can get caught out if we do not test our assumptions.

\section{Rule out worst case scenarios}

This approach focuses on risk management but can often leave our diagnostic question unanswered. For example, with acute chest pain we may exclude many serious differentials but fail to reach a definite diagnosis.

\section{Casablanca strategy}

The Casablanca strategy is to 'round up the usual suspects.'. ${ }^{11}$ It represents the lazy application of a standard battery of tests for a given symptom and is somewhat akin to working on autopilot. ${ }^{12}$

\section{Hypothesis testing and reflection}

Malcolm Gladwell's bestselling book Blink: the power of thinking without thinking popularised the concept of rapid, intuitive decision making with its strengths and weaknesses. ${ }^{13}$ Thinking fast and slow by Daniel Kahneman further explores and explains this two-system approach to judgment and choice. ${ }^{14}$ System 1 is fast, automatic, emotional, stereotypic, used frequently and operates subconsciously. System 2 is slow, effortful, logical, calculating, used infrequently and requires conscious thought. In most circumstances, we are better served by following a system 2 approach. Strangely, the decision as to which system to use rests with system 1 , and when we are under pressure, we default to system 1 thinking. The dual system model is also applicable to medical decision making. ${ }^{15,16}$

The first step involves data gathering from the history and examination (Fig 1a); this stage also incorporates assessing test results. This information forms the basis for hypothesis generation. At this point, pattern recognition, rule out worst case scenarios, and even the Casablanca strategy come into play. These hypothesis-generating steps are not enough: we must examine them, refute them if possible and then attempt to also find confirmatory data where it can be provided. This whole process is the involved, deliberate, analytical, effortful endeavour of system 2 thinking. We may be tempted to short circuit the process in a variety of ways. We may stop if we think we recognise a familiar pattern. We may be happy if there is a positive test result, even if it does not fit in with the history. Heuristics are mental shortcuts that can aid rapid decision making, and are an example of system 1 thinking that experienced thinkers may use to bypass the laborious process of deliberate thought. This approach is not without risk. An example where the patient history seems to fit a recognisable pattern and, therefore, the clinician has given little consideration to other sources of information thus neglecting other potential alternative diagnostic hypotheses can be seen in Fig $1 \mathrm{~b}$. The clinician jumps straight to confirmatory investigations without truly testing his hypothesis. If this works, it may seem like diagnostic brilliance but there is great potential for failure. Heuristics are often accompanied by biases based on our cognitive disposition to respond (CDR).
A

Data gathering

History, examination, screening tests, eg ECG bloods

$\downarrow$

Hypothesis generation

Patterns recognition, ROWS,

Casablanca strategy

$\downarrow$

Hypothesis testing and confirmatory testing

D-dimer, troponin CT scan, pathology

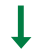

Reflection/explanation

$\downarrow$

Diagnosis
B

Data gathering

History, examination, screening tests

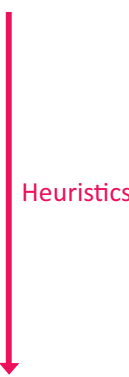

Diagnosis

\section{C}

History and examination

Faulty data gathering

- slips and lapses

Hypothesis generation

Faulty hypothesis generation

$\downarrow$ • knowledge-based mistakes Hypothesis testing

Faulty hypothesis testing

$\downarrow$ - rule-based mistakes

Reflection/explanation

Faulty reflection

- rule-based mistakes/

knowledge-based

$\downarrow$ mistakes/biases

Diagnosis (possibly incorrect)

Fig 1. The process of medical decision making. A - diagnosic process; B - heuristic thinking bypasses reasoned thought; C - errors are possible at each stage of the process. 


\begin{tabular}{lll} 
A & \multicolumn{2}{c}{ Syllogisms } \\
\hline Modus ponens & Modus tolens & Disjunctive syllogism \\
$\begin{array}{lll}\text { 1. If } P \text { then } Q & \text { 1. If } P \text { then } Q & \text { 1. Either } P \text { or } Q \\
\text { 2. } P & \text { 2. Not } Q & \text { 2. Not } P \\
\text { 3. Therefore, } Q & \text { 3. Therefore, not } P & \text { 3. Therefore, } Q\end{array}$
\end{tabular}

\begin{tabular}{|c|c|}
\hline \multicolumn{2}{|c|}{ Examples } \\
\hline Modus ponens & Modus tolens \\
\hline $\begin{array}{l}\text { 1. If SAH then } \\
\text { xanthochromia } \\
\text { on CSF }\end{array}$ & $\begin{array}{l}\text { 1. If SAH then } \\
\text { xanthochromia } \\
\text { on CSF }\end{array}$ \\
\hline 2. SAH & 2. No xanthochromia \\
\hline $\begin{array}{l}\text { 3. Therefore, } \\
\text { xanthochromia }\end{array}$ & 3. Therefore, no SAH \\
\hline
\end{tabular}

\author{
C Fallacies \\ Modus ponens \\ 1. If $P$ then $Q$ \\ 2. $P$ Q \\ 3. Therefore, $Q P$ \\ Affirming the consequence \\ 1. If PE then D-dimer is raised \\ 2. D-dimer is raised \\ 3. Therefore, PE
}

Fig 2. Modus ponens (mode of putting), modus tolens (mode of taking) and disjunctive syllogisms. A - the structure of syllogisms; B - examples of syllogisms; C - an example of a common fallacy. CSF = cerebrospinal fluid; $\mathrm{PE}=$ pulmonary embolism; $\mathrm{SAH}=$ subarachnoid haemorrhage.

\section{Where thinking goes wrong}

As seen in Fig 1c, errors can occur any at stage. Psychologist James Reason identifies three basic error types: skill-based (slips and lapses), rule-based and knowledge-based. ${ }^{17}$ Skillbased errors may occur because we get distracted and follow a habitual course of action rather than our original plan, perhaps intending to follow up on a trainee's comments about a murmur but instead finding ourselves in the usual routine of checking blood results and radiology reports. The frequent interruptions and distractions of the ward environment mean this is not unusual. Rule-based mistakes include the misapplication of a good rule or the application of a bad rule. One such rule that is often misapplied is that of Occam's razor, which states that 'entities must not be multiplied beyond necessity. ${ }^{18}$ However, one must not ruthlessly discount valid alternatives; to apply another rule, 'patients can have as many diseases as they damn well please' (Hickam's dictam). ${ }^{19}$

Knowledge-based mistakes can include lack of appropriate information, but can also arise through a lack of understanding of the principles of logic. While diagnostic reasoning is not purely deduction, we must be able to think logically. Let us for a moment consider the use of syllogisms. ${ }^{20}$ These are a kind of argument using deductive reasoning, based on the relationship of two or more propositions that are assumed, or asserted, to be true. There are three forms: modus ponens (mode of putting), modus tolens (mode of taking) and disjunctive syllogisms (Fig 2).

At this point, it is worth considering a logical distinction between necessary and sufficient causes (Fig 3). To quote Carl Trueman again 'a necessary cause is such that, if phenomenon $\mathrm{B}$ is present, then A must be present too; though the presence of A does not necessarily imply that B will occur... a sufficient cause is such that A necessarily implies the presence of $B$, but $B$ could be caused by $C$; thus the presence of $B$ does not mean that $\mathrm{A}$ is necessarily present'. ${ }^{8}$ To make a diagnosis, we are seeking the necessary and sufficient explanation for the patient's condition; what lawyers term the 'necessary elements of a sufficient set'.

\section{Bias and cognitive disposition to respond}

Errors can arise when we allow biases to distort our thoughts. These biases can also be referred to as our CDR. Croskerry lists a considerable number of potential CDRs. ${ }^{10}$ Order effects mean that in receiving information, we pay more attention at the beginning and end of a story; meaningful detail in the middle

can get lost, especially if we do not interrogate all the initial information. We may fall prey to the availability heuristic, judging things to be more likely if they readily come to mind, or we may follow Sutton's law and look 'where the money is' (named after Willie Sutton, New York bank robber). Anchoring means that we lock into salient features too early. This then allows the development of diagnosis momentum: once a label is attached, it becomes increasingly sticky. With confirmation bias causing us to look for evidence to support our initial diagnosis, search satisfying means calling off the search early, potentially missing co-pathology. This all results in premature closure (accepting a diagnosis before it has been verified). ${ }^{10}$

Personal factors also come into play. Overconfidence means we act on a hunch with incomplete information, relying on opinion instead of evidence. Also, there are the sunk costs of following a certain line of thought: time, energy and ego. We may exhibit social bias in the fundamental attribution error of blaming the patient for their diseases, or we may allow our softer side to favour diagnoses with better outcomes - so-called outcome bias.

A lack of statistical awareness can skew our thinking. Base rate neglect means we ignore the true prevalence of disease, inflating or neglecting its base rate. The gambler's fallacy means we are unduly influenced by preceding events rather than the true probability of an individual occurrence ('four subarachnoid haemorrhages in a row? This one must be migraine'). The opposite of this is posterior probability error - just because you have a migraine, doesn't mean you can't have a subarachnoid bleed.

\section{Making better decisions}

\section{Preparing ourselves}

Before we consider our reasoning, we must focus on the task at hand. Mindfulness is a concept often associated with relaxation or stress management. However, it is also relevant to our discussion. We must avoid working on autopilot, ${ }^{12}$ give our full attention to each patient in turn and not allow ourselves to be

For pneumonia...

Necessary but not sufficient
- air space shadowing on chest
X-ray

Fig 3. Necessary elements of a sufficient set.
Necessary and sufficient

- air space shadowing on chest X-ray, plus

- clinical signs of chest sepsis 
sidetracked by distractions around us or rumination about the cases just seen. We must also take account of the impact of our mood and our environment.

\section{Data gathering}

We have already considered the importance of gathering accurate information when taking a history. Even if our thought processes are optimal, there is a high possibility that an error will be made if the limitations of the data that we interpret are not appreciated. The following brief discussion focuses on laboratory data, but some of the principles equally apply to other tests.

First, there is always the possibility of a pre-analytical error. This incorporates issues such as:

$>$ mislabelling of a blood sample

$>$ inappropriate collection of a sample, eg blood being drawn from a 'drip arm'

$>$ a technical issue with the sample, eg an air bubble in a blood gas syringe.

Analytical errors occur when things go wrong in the laboratory. Post-analytical errors are in the domain of the physician interpreting the test, and range from simple (eg transcription errors) to more complex (eg incorrect interpretation of a result due to a gap in knowledge). Reference ranges for parameters are often constructed in such a way that they will encapsulate results from $95 \%$ of healthy individuals. $5 \%$ of healthy people will therefore have an 'abnormal' result that is of no pathological significance. Similarly, a proportion of patients with disease will have a seemingly normal result. A correct comparison of a test result with a previous one should consider biological variability and imprecision in the laboratory. Knowledge about sensitivity, specificity and positive/negative predictive values of a test can be used to reach diagnostic conclusions with more certainty.

\section{Thinking about thinking}

Once we have the relevant information regarding the case, how do we safeguard our thinking processes? We need to have insight into the problems and be aware of how we think (metacognition). Then, as we make decisions, we need to reflect on the decision-making process, to be aware of biases and rushed system 1 errors. We can aim to develop our own clinical reasoning from the unreflective to that of the accomplished thinker. ${ }^{16}$ We can use cognitive forcing strategies, such as structured review of data and diagnostic checklists. ${ }^{22}$ We can also use resources to decrease dependence on memory. We can make ourselves accountable to the rest of the team for our thinking processes. 'Thinking out loud' on our ward rounds will allow others to follow our train of thought and permit challenge when our reasoning is flawed. Training tools, such as the One Minute Preceptor, can help our trainees develop thinking skills (and keep us fresh as we supervise them). ${ }^{23}$ It is important too that we develop skills in error recognition and recovery. ${ }^{24}$

Lastly, we return to the third of Pellegrino's questions: what is the best solution for this patient? ${ }^{4}$ Please excuse the pun, but the thinking doctor should also be the thoughtful doctor. Ballat and Campling prompt us to be attentive to the whole needs of the patient, to exhibit intelligent kindness, or, as has been said elsewhere, 'one of the essential qualities of the clinician is interest in humanity, for the secret of the care of the patient is in caring for the patient'. ${ }^{25,26}$

\section{Conflicts of interest}

The authors declare no competing interests.

\section{References}

1 Trimble M. The thinking doctor's ward round. Ulster Med J $2015 ; 84 ; 3-7$.

2 Kohn L, Corrigan J, Donaldson M (Eds.) To err is human: building a safer health system. Washington, D.C.: National Academy Press, 2000.

3 Leape L, Brennan T, Laird N et al. The nature of adverse events in hospitalized patients - results of the Harvard Medical Practice Study II. N Engl J Med 1991;324;377-84.

4 Pellegrino E, Thomasma D. A philosophical basis of medical practice: towards a philosophy and ethic of the healing professions. Oxford: Oxford University Press, 1980.

5 Schleifer R, Vannatta J. The logic of diagnosis: peirce, literary narrative, and the history of present illness. J Med Philos 2006;31;363-84.

6 Rapezzi C, Ferrari R, Branzi A. White coats and fingerprints: diagnostic reasoning in medicine and investigative methods of fictional detectives. Brit Med J 2005;331;1491-4.

7 Conan Doyle A. A scandal in Bohemia. London: Strand Magazine, 1891.

8 Trueman C. Histories and fallacies: problems faced in the writing of history. Illinois, Crossway, 2010.

9 Conan Doyle A. The Penguin Complete Sherlock Holmes. London: Penguin Books, 1981.

10 Croskerry P. Achieving quality in clinical decision making: cognitive strategies and detection of bias. Acad Emerg Med 2002;9;1184-204.

11 Attributed to Captain Renault, Casablanca, Warner Bros., 1942.

12 Williams M, Penman D. Mindfulness: a practical guide to finding peace in a frantic world. London: Piatkus, 2011.

13 Gladwell M. Blink: the power of thinking without thinking. London: Little, Brown and Company, 2005.

14 Kahneman D. Thinking, fast and slow. London: Penguin Books, 2012

15 Croskerry P. Clinical cognition and diagnostic error: applications of a dual process model of reasoning. Adv Health Sci Educ Theory Pract 2009;14(Suppl 1);27-35.

16 Croskerry P, Nimmo G. Better clinical decision making and reducing diagnostic error. J R Coll Physicians Edinb 2011;41;155-62.

17 Reason J. Human Error. Cambridge: Cambridge University Press, 1990.

18 Attributed to William of Ockham, philosopher, c. 1285-1349.

19 Attributed to Hickam J, Chairman of Medicine, Indiana University.

20 Weston A. A rulebook for arguments, 3rd edn. Indianapolis: Hackett Publishing Company, Inc., 2000.

21 Wright R. Once more into the bramble bush: duty, causal contribution and the extent of legal responsibility. Vanderbilt Law Rev 2001;53;1071-131.

22 Neher J, Gordon K, Meyer B, Stevens N. A five-step microskills model of clinical teaching. J Am Board Fam Pract 1992;5;419-24.

23 Ely J, Graber M, Croskerry P. Checklists to reduce diagnostic errors. Acad Med 2011;86;307-13.

24 Dror I. A novel approach to minimize error in the medical domain: cognitive neuroscientific insights into training. Med Teach 2011;33;34-8.

25 Attributed to Peabody FW (1881-1927), Professor of Medicine, Harvard Medical School

26 Ballatt J, Campling P. Intelligent kindness. London: RCPsych, 2011.

Address for correspondence: Dr P Hamilton, Belfast Health and Social Care Trust, Kelvin Building, Royal Hospitals, Grosvenor Road, Belfast BT12 6BA, UK.

Email: paul.hamilton@belfasttrust.hscni.net 